Pacific Journal of Mathematics

THE COUPLED YANG-MILLS-DIRAC EQUATIONS FOR 


\title{
THE COUPLED YANG-MILLS-DIRAC EQUATIONS FOR DIFFERENTIAL FORMS
}

\author{
Thomas H. OTWAY
}

\begin{abstract}
A version of the coupled Yang-Mills-Dirac equations for differential forms is presented. In this version the equations are defined and conformal in any odd dimension; they share many of the analytic properties of the Yang-Mills-Higgs equations in these dimensions. A point singularity problem is formulated and solved for the YangMills-Dirac equations in dimension 3. In this dimension the solutions can be associated with a definite energy functional resembling the magnetic-monopole energy.
\end{abstract}

1. The Yang-Mills-Dirac equations are a coupled system of nonlinear partial differential equations in which the unknowns are sections of twisted vector bundles. When the base space is $\mathbb{R}^{4}$ these equations can be used to describe the interaction of an external force field (the Yang-Mills field $F_{A}$ ) with the field $\varphi$ induced by a fermion (the Dirac field).

In dimensions $n \neq 4$ the equations have mathematical interest as an example of a system which is elliptic modulo the action of a symmetry group. That is, let $(A, \varphi)$ be a solution of the Yang-Mills-Dirac equations and let $G$ be a fixed transformation group. Then the pair $(g(A), g(\varphi)), g \in G$, is also a solution and we identify $(A, \varphi)$ with $(g(A), g(\varphi))$. There is a $g_{0} \in G$ such that $\left(g_{0}(A), g_{0}(\varphi)\right)$ is a solution of an elliptic system of partial differential equations, although the system satisfied by $(A, \varphi)$ may not be elliptic.

In such a model the removability of singularities is a particularly interesting problem for two reasons. First, the Yang-Mills-Dirac equations have an interpretation as the Euler-Lagrange equations of an energy functional, so ordinarily one would consider weak solutions and show the existence of classical solutions by a partial regularity argument. Unfortunately, the concept of a weak solution is ambiguous in this case, since $(A, \varphi)$ and $(g(A), g(\varphi))$ need not lie in the same function spaces. Thus one is motivated to try to characterize the singular set by asking what kind of singularities a classical solution can have. Second, the transformation $g_{0}$ may act discontinuously on the fiber, changing the topology of the vector bundle and thus altering the 
original singular set. If we want to use elliptic theory in order to remove singularities by applying transformations in $G$, we will therefore want to choose among continuous elements of $G$ in order to preserve the singular set. The obstruction to doing this is the smoothness of the Yang-Mills field $F_{A}$, which is represented by the bundle curvature. Precisely, the transformation $g_{0}$ is guaranteed continuous if and only if $F_{A}$ lies in the space $L^{p}$ for some $p>n / 2$ in a region about the singularity [11].

T. H. Parker showed [4] that solutions cannot have point singularities in dimension 4 if the total energy of the coupled field is finite. Conditions for the nonexistence of point singularities in (even) dimensions $n \geq 4$ were given in [3]. In dimension 2 the Dirac equation reduces to the eigenvalue equation for a twisted Cauchy-Riemann operator. Point singularities in this dimension can be removed by an argument similar to [9], provided $F_{A} \in L^{1}$ and $\varphi \in H^{1,2}$ at the puncture and provided that a holonomy condition is assumed. All these arguments require techniques developed by $\mathrm{K}$. Uhlenbeck for the pure Yang-Mills equations in dimension 4 ([10], [11]).

The close mathematical relation between solutions of the YangMills-Dirac equations and those of the Yang-Mills-Higgs equations suggests that the point singularity problem should have interesting features in dimension 3. In this dimension the corresponding problem for the Yang-Mills-Higgs equations requires delicate analytic techniques ([6], [8]).

In fact the Yang-Mills-Dirac equations are of particular interest in that, in distinction to the Yang-Mills-Higgs equations, in the former system $\varphi$ is coupled to $F_{A}$ in both (second-order) equations [see eqs. (1.2)]. Thus an improvement in the smoothness of $\varphi$ does not give an immediate improvement in the smoothness of its coefficients.

In $n=2 k$ dimensions the Yang-Mills-Dirac equations can be written as a first-order system in $\left(F_{A}, \varphi\right)$ :

$$
\left\{\begin{array}{l}
D_{A}^{*} F_{A}=J(\varphi) \\
\not_{A} \varphi=m \varphi
\end{array}\right.
$$

Here $D_{A}^{*}: \Lambda^{p}(\operatorname{ad} E) \rightarrow \Lambda^{p-1}(\operatorname{ad} E)$ is the formal adjoint of the exterior covariant derivative $D_{A}$ on a vector bundle $E$ with connection 1-form $A$; the lie-algebra-valued 2 -form $F_{A}$ is a local representation of the curvature of $E$; the spinor $\varphi$ is a smooth section of $V \otimes E$, where $V$ is a $2 k$-dimensional complex vector space isomorphic to the Clifford algebra $C(E)$ of $E ; \not_{A}$ is the covariant Dirac operator on twisted spin bundles; $m$ is a section of a trivial real line bundle. If 
$E$ is derived from a principal bundle with compact structure group $G$ and $\rho$ is a unitary representation of $G$, then

$$
J(\varphi)=-\frac{1}{2}\left\langle\varphi, e^{i} \cdot \rho\left(\sigma^{\alpha}\right) \varphi\right\rangle \sigma_{\alpha} \otimes e_{i},
$$

where $\langle$,$\rangle denotes inner product, \sigma^{\alpha}$ is an orthogonal basis for the lie algebra of $G$, and $\left\{e_{i}\right\}$ is an orthonormal frame. (See [4] for details.)

In odd dimensions it is customary [1] to replace $\not_{A}$ by the operator $e_{2 k} \not \partial_{A}$, where $e_{1}, \ldots, e_{2 k}$ is a basis for $C(E)$. This entails a restriction on the sign of $m$; whereas the eigenvalues of $\not_{A}$ are symmetric about the origin, the eigenvalues of $e_{2 k} \not_{A}$ lie on a half-axis. No restriction on the sign of $m$ occurs if we use the vector-space isomorphism between $C(E)$ and $\Lambda^{*}(E)$ to replace the Dirac equation in (1.1) by a second-order equation on 1-forms; taking $n=3$ we have, denoting by $\underline{D}_{A}$ the total covariant derivative on $\Lambda^{*} \otimes E$,

$$
\left\{\begin{array}{l}
D_{A} * F_{A}=\frac{1}{2}[\varphi, \varphi], \\
\not_{A}^{2} \varphi=\underline{D}_{A}^{*} \underline{D}_{A} \varphi+\frac{1}{2} \sum_{i, j}^{n=3} e^{i} \cdot e^{j} \cdot F_{i j}(\varphi)=m^{2} \varphi .
\end{array}\right.
$$

Here $\left\{e_{i}\right\}$ is an orthonormal basis of 1 -forms; $\varphi \in \Lambda^{1}(\operatorname{ad} E) ;[$, ] denotes lie bracket; $*: \Lambda^{p} \rightarrow \Lambda^{n-p}$ is the Hodge involution.

The motivation for writing eqs. (1.1) in the form (1.2) comes from the theory of conformal invariance. One wants to determine a choice for the conformal weight $w$ of a solution $\varphi$ to eqs. (1.1) that will be meaningful in odd dimensions.

Recall that a section $\psi$ of a (weightless) vector bundle is said to have conformal weight $w$ if for any scalar $e^{\lambda}$

$$
\left|\psi\left(e^{\lambda} x\right)\right|=e^{-\lambda w}|\psi(x)|
$$

Classically, $\psi$ is written as a weight- $w$ relative tensor: under coordinate transformations $x \rightarrow y$,

$$
\tilde{\psi}_{I}(y)=\left|\frac{\partial x}{\partial y}\right|^{w} \psi_{I}(x) ;
$$

globally $\psi$ is a section of the tensor product of the determinant bundle raised to the power $w / n$ and a weightless vector bundle.

For example, a $p$-form always has conformal weight $p$ due to the invariance of differential forms under coordinate transformations. Let $\alpha(x)=a_{i_{1} \cdots i_{p}}(x) d x^{i_{1}} \wedge \cdots \wedge d x^{i_{p}}$. Since $\alpha$ is a section of a vector bundle it must satisfy $\alpha\left(e^{\lambda} x\right)=\alpha(x)$ in order to avoid coordinate 
dependence. Thus

$$
\begin{aligned}
\alpha\left(e^{\lambda} x\right) & =\tilde{a}_{i_{1} \cdots i_{p}}\left(e^{\lambda} x\right) e^{p \lambda} d x^{i_{1}} \wedge \cdots \wedge d x^{i_{p}} \\
& =\alpha(x)=a_{i_{1} \cdots i_{p}}(x) d x^{i_{1}} \wedge \cdots \wedge d x^{i_{p}} .
\end{aligned}
$$

This can only occur if

$$
\tilde{a}_{i_{1} \cdots i_{p}}\left(e^{\lambda} x\right)=e^{-p \lambda} a_{i_{1} \cdots i_{p}}(x) .
$$

(See [4] and [5] for a general treatment.)

In dimension 4 it is necessary to choose $w=3 / 2$ so that the coupled spinor Lagrangian

$$
\mathscr{L}^{4}(x)=\int_{\mathbb{R}^{4}}\left\{\left|F_{A}\right|^{2}+\left\langle\varphi, \not_{A} \varphi\right\rangle\right\} d^{4} x
$$

will be invariant under conformal transformations (coordinate changes of the form $y=e^{\lambda} x$ ). Since $F_{A}$ is a 2 -form and thus has conformal weight 2 , and since any differential operator increases conformal weights by 1 (by the chain rule), this Lagrangian is conformally homogeneous in any dimension if $w=3 / 2$ :

$$
\mathscr{L}^{n}(r x)=r^{n-4} \mathscr{L}^{n}(x), \quad r>0 .
$$

For this reason the conformal weight of $\varphi$ was chosen to be $3 / 2$ in [3].

If $n=4$, then the field equations (1.1) are the variational equations for $\mathscr{L}^{4}(x)$ when $m=0$. However, this variational structure is relatively unimportant mathematically; this is due to the fact that $\mathscr{L}^{4}(x)$ is not bounded below as a functional as a result of the negative eigenvalues of $\not_{A}$. There is, however, value in a conformally invariant energy functional since it can be made small, again provided that it is definite [cf. (2.3), below]. Thus we seek a conformally invariant "energy" functional without requiring that it be related variationally to solutions of (1.1) or (1. '). The obvious choice,

$$
\int_{\mathbb{R}^{n}}\left\{\left|F_{A}\right|^{n / 2}+\left\langle\varphi, \not_{A} \varphi\right\rangle\right\} d^{n} x
$$

with $w=(n-1) / 2$, is rejected since it is not bounded below. However, if $n=3$ there is a very simple candidate. If the total field $h(x)$ is defined to be

$$
h=\left|F_{A}\right|+\left|\underline{D}_{A} \varphi\right|+|\varphi|^{2}
$$

(cf. [4] and [8]) with $w=(n-1) / 2=1$ the conformal weight of $\varphi$, then the energy functional 
is bounded below by zero, conformally homogeneous for any $p$, and invariant if $p=n / 2$.

Notice that if $n=4$, then $w=(n-1) / 2=3 / 2$. In this sense our choice of conformal weight is a generalization of the 4-dimensional case. Further, in odd dimensions $\varphi$ scales like an $(n-1) / 2$-form. In particular, if $n=3$ then $\varphi$ scales like a 1-form (i.e., like a boson). The second of eqs. (1.2) is just the Weitzenböck formula for the Dirac operator on $E$-valued 1 -forms (eq. (1.18) of [4]). The first-order Dirac equation given in (1.1) is in fact a conformally invariant expression in $n$ dimensions precisely when $w=(n-1) / 2$ (see p. 11 of [1] and eq. (3.1) of [4]).

As for the first of eqs. (1.2), if $\varphi \in \Lambda^{(n-1) / 2}(\operatorname{ad} E)$ for $n$ odd, then

$$
*\left(\frac{1}{2}[\varphi, \varphi]\right)=* \varphi \wedge \varphi \in \Lambda^{1}(\operatorname{ad} E) .
$$

Thus applying the Hodge operator to the first of eqs. (1.2) gives a form of the coupled Yang-Mills equations

$$
D_{A}^{*} F_{A}=J(\varphi)
$$

where the "current" $J(\varphi)$ is given by (1.4).

Solutions of systems (1.1) or (1.2) satisfy the following subelliptic inequalities

$$
\left\{\begin{array}{l}
\Delta\left|F_{A}\right|+C_{0}\left(\left|F_{A}\right|^{2}+|\varphi|\left|D_{A} \varphi\right|\right) \geq 0, \\
\Delta|\varphi|+C_{1}\left|F_{A}\right||\varphi| \geq 0
\end{array}\right.
$$

Such solutions are assumed to be invariant under the action of $G$ valued maps. These maps act tensorally on $F_{A}$ and $\varphi$ but affinely on the connection 1 -form $A$. If

$$
F=d A+\frac{1}{2}[A, A],
$$

then

$$
g(F)=g^{-1} F g \quad \text { and } \quad g(\varphi)=g^{-1} \varphi g
$$

but

$$
g(A)=g^{-1} A g+g^{-1} d g, \quad g \in G .
$$

Such maps are called gauge transformations.

Denote by $B$ the unit ball centered at the origin of coordinates in $\mathbb{R}^{n}$. The point singularity problem in gauge theory involves finding conditions under which a $C^{\infty}$ solution of the field equations in $B-$ $\{0\}$ extends to a $C^{\infty}$ solution in $B$. The extension is effected via a continuous (topology-preserving) gauge transformation [10].

Notice that if $w=(n-1) / 2$, a version of (1.2) exists for any odd $n$, although (1.3) is not useful unless $n=3$. Since we do not use 
the indefinite Lagrangian $\mathscr{L}^{4}$, and since the eigenvalues of $\not_{A}^{2}$ are nonnegative, we do not expect singularities in eqs. (1.2) to "integrate out" (cf. $\S \S 2$ and 3 of [4]). Thus we prove:

THeOREM 1.1A. Let $F_{A} \in L^{n / 2}(B)$ and $\varphi \in L^{p}(B)$ for some $p>$ $n /(n-2)$, where $B$ is an $n$-dimensional ball about the origin of coordinates in $\mathbb{R}^{n}, n=2 k+1, k \in \mathbb{Z}^{+}-\{1\}$. Let $\varphi \in \Lambda^{(n-1) / 2}(\operatorname{ad} E)$ and $F_{A} \in \Lambda^{2}($ end $E)$ satisfy in $B-\{0\}$ the equations

$$
\left\{\begin{array}{l}
D_{A} * F_{A}=\frac{1}{2}[\varphi, \varphi], \\
D_{A}^{*} D_{A} \varphi+\frac{1}{2} \sum_{i, j}^{n} e^{i} \cdot e^{j} \cdot F_{i j}(\varphi)=m^{2} \varphi .
\end{array}\right.
$$

Then $\left(F_{A}, \varphi\right)$ is equivalent via a continuous gauge transformation to a $C^{\infty}$ solution in $B$.

The proof of Theorem 1.1A requires that Lemma 2.1 of the following section be applied to $\varphi, D_{A} \varphi$, and $F_{A}$. In the latter two applications the hypothesis $q_{0}>1 / 2$ of the lemma restricts its application to the case $n>4$. Thus the following 3-dimensional theorem requires a different proof than the higher-dimensional case:

Theorem 1.1B. Let $\left(F_{A}, \varphi\right)$ be a $C^{\infty}$ solution of eqs. (1.2) in $B-$ $\{0\}$. Suppose that $F_{A} \in L^{3 / 2}(B)$ and $\varphi \in L^{p}(B)$ for some $p>3$. Then $\left(F_{A}, \varphi\right)$ is equivalent via a continuous gauge transformation to a $C^{\infty}$ solution in $B$.

2. We now prove the results stated in the previous section. We denote by $C$ positive constants the value of which may change from line to line.

We require the following result from elliptic theory:

LemMA 2.1 [7]. Let $u(x) \geq 0$ be $C^{\infty}$ in $B-\{0\}$ and satisfy there

$$
\Delta u+f(x) u \geq 0
$$

for $f \in L^{n / 2}(B)$. If for $1 / 2<q_{0}<q$ we have $u \in L^{2 n q_{0} /(n-2)} \cap L^{2 q}(B)$, then $\nabla\left(u^{q}\right) \in L^{2}(B)$ and in a sufficiently small ball $\widetilde{B}, \forall \eta \in C_{0}^{\infty}(\widetilde{B})$ $u$ satisfies

$$
\int_{\widetilde{B}} \eta^{2}\left|\nabla u^{q}\right|^{2} d x \leq C \int_{\widetilde{B}}|\nabla \eta|^{2} u^{2 q} d x
$$

Notice that the test function $\eta$ in Lemma 2.1 is not required to vanish in a neighborhood of the puncture. 
We begin by proving Theorem 1.1A.

Apply Lemma 2.1 with $u=|\varphi|, f=C|F|, q_{0}=1 / 2+\varepsilon$, and $q=n / 2(n-2)+\varepsilon$. We conclude that $\nabla u^{q}$ is in $L^{2}(B)$ and apply Sobolev's inequality to obtain a better $L^{p}$ estimate for $|\varphi|$. Iterating the lemma with new values for $q_{0}$ and $q$ we find in a finite number of steps that $\varphi \in L^{r}(B)$ for any finite value of $r$.

LEMMA 2.2. Under the hypotheses of Theorem 1.1A, $D_{A} \varphi \in L^{2}(B)$.

Proof. Integrate $D_{A} \varphi$ by parts against $D_{A} \xi$, where $\xi=(\eta \bar{\eta})^{2} \varphi$, $0 \leq \eta \leq 1,0 \leq \bar{\eta} \leq 1, \bar{\eta}(x)=0 \forall x:|x| \leq \delta$. (In fact $\eta$ and $\bar{\eta}$ can both be chosen to be radial functions.) We obtain from the Young and Hölder inequalities

$$
\begin{aligned}
\int_{B}\left|D_{A} \varphi\right|^{2}(\eta \bar{\eta})^{2} d x \leq & 2 \varepsilon \int_{B}\left|D_{A} \varphi\right|^{2}(\eta \bar{\eta})^{2} d x \\
& +2 \varepsilon^{-1} \int_{B}|\nabla \eta \bar{\eta}+\eta \nabla \bar{\eta}|^{2}|\varphi|^{2} d x \\
& +\left\|F_{A}\right\|_{L^{n / 2}(B)}\|\varphi\|_{L^{2 n /(n-2)}(B)}^{2}
\end{aligned}
$$

We can choose $\bar{\eta}=\bar{\eta}^{(\delta)}$ so that $\bar{\eta}^{(\delta)} \rightarrow 1$ and $\left\|\nabla \bar{\eta}^{(\delta)}\right\|_{L^{n}} \rightarrow 0$ as $\delta \rightarrow 0$ [7]. We find that in the limit

$$
\begin{aligned}
(1-2 \varepsilon) & \int_{B}\left|D_{A} \varphi\right|^{2} \eta^{2} d x \\
\leq & 2 / \varepsilon\left(\int_{B}|\nabla \eta|^{2} \varphi^{2} d x+\lim _{\delta \rightarrow 0}\left\|\nabla \bar{\eta}^{(\delta)}\right\|_{L^{n}(B)}^{2}\|\varphi\|_{L^{2 n /(n-2)}(B)}^{2}\right) \\
& +\left\|F_{A}\right\|_{L^{n / 2}(B)}\|\varphi\|_{L^{2 n /(n-2)}(B)}^{2}<\infty .
\end{aligned}
$$

Choosing $\eta(x)=1$ for $x \in \widetilde{B} \subset B$ completes the proof of Lemma 2.2.

Notice that the scalar $\psi=\left|D_{A} \varphi\right|+1$ satisfies

$$
\Delta \psi+C\left(|\varphi|^{2}+\left|F_{A}\right|\right) \psi \geq 0
$$

in $B-\{0\}$. Apply Lemma 2.1 with $u=\psi, f=C\left(|\varphi|^{2}+\left|F_{A}\right|\right)$, $q_{0}=(n-2) / n$, and $q=1$. The hypotheses of the lemma are satisfied provided $n>4$. Reasoning exactly as in the case $u=|\varphi|$ we conclude that $D_{A} \varphi \in L^{r}(B)$ for any finite $r$.

The scalar function $v=|F|+1$ satisfies

$$
\Delta v+C\left(|F|+|\varphi|\left|D_{A} \varphi\right|\right) v \geq 0
$$


in $B-\{0\}$. Apply Lemma 2.1 with $u=v, f=C\left(|F|+|\varphi|\left|D_{A} \varphi\right|\right)$, $q_{0}=(n-2) / 4$, and $q=n / 4$. Again we find that the conditions of the lemma are met whenever $n$ exceeds 4 . We conclude that $F \in L^{r}(B)$ for any finite $r$.

Making a continuous gauge transformation to a gauge in which $A$ is a weak solution of an elliptic system, we easily complete the proof by the methods in Chapter 5 of [2].

We now prove Theorem 1.1B.

LEMMA 2.3. The curvature $F_{A}$ is in $L^{p}(B)$ for $3 / 2<p<3 /(2-\delta)$, $\delta>0$, and $\left(F_{A}, \varphi\right)$ is a weak solution of (1.2) in all of $B$.

Proof. We argue as in $\S \S 3$ and 4 of [8]. Defining $h$ as in (1.3) we observe that inequalities (1.5) and (2.1) imply that $h$ satisfies

$$
\Delta h+C h^{2} \geq 0
$$

in $B-\{0\}$.

Decompose $B-\{0\}$ into a countable union of 3-dimensional annuli $V_{\rho}=\{x|\rho / 2 \leq| x \mid \leq \rho\}, 0<\rho \leq 1$. The conformal dilation $y=$ $\rho^{-1} x$ carries $V_{\rho}$ into the "reference" annulus $V=\{y|1 / 2 \leq| y \mid \leq 1\}$. Both the field equations (1.2) and the norm $\|h\|_{L^{3 / 2}(B)}$ are invariant under this dilation.

Let $y_{0}$ be a point on the sphere $|y|=3 / 4$. Integrate $\nabla h$ by parts against $\nabla \xi$, where

$$
\xi=\eta^{2} h^{1 / 2}, \quad \eta \in C_{0}^{\infty}\left(B_{\lambda}\left(y_{0}\right)\right),
$$

for $\lambda$ a sufficiently small positive number. By Young's inequality,

$$
\int_{B_{\lambda}\left(y_{0}\right)}\left|\nabla h^{3 / 4}\right|^{2} d y \leq C a^{-2} \int_{B_{\lambda+a}\left(y_{0}\right)} h^{3 / 2} d y
$$

provided $\|h\|_{L^{3 / 2}\left(B_{\lambda}\left(y_{0}\right)\right)}<\gamma_{0}$. (This can always be arranged due to the conformal invariance of the field equations and of this norm; see Lemma 1.1 of [8].)

Using (2.2), we can apply Theorem 5.3 .1 of [2] with $U=h^{3 / 4}$, $w=U^{4 / 3}$ to conclude that

$$
h(y) \leq C\|h\|_{L^{3 / 2}(1 / 2 \leq|y| \leq 1)} .
$$

Scaling this inequality we obtain

$$
|x|^{2} h(x) \leq C\|h\|_{L^{3 / 2}(\rho / 2 \leq|x| \leq \rho)}<\gamma .
$$


We can apply Uhlenbeck's broken Hodge gauge construction [10] using weighted norms as in [8]. Write $B-\{0\}=\bigcup_{i=1}^{\infty} U_{i}$, where $U_{i}$ are 3-dimensional annuli centered at the origin: $U_{i}=\left\{x\left|1 / \tau^{i} \leq\right| x \mid \leq\right.$ $\left.1 / \tau^{i-1}\right\}, 1<\tau<2$. Then

$$
\begin{aligned}
\int_{U_{l}}|x|^{\alpha}\left|F_{A}^{i}(x)\right|^{2} d x= & \int_{U_{i}}\left\langle A^{i}, D_{A}^{*}\left(|x|^{\alpha} F_{A}^{i}\right)\right\rangle d x \\
& -\int_{U_{l}}\left\langle\frac{1}{2}\left[A^{i}, A^{i}\right],|x|^{\alpha} F_{A}^{i}\right\rangle d x \\
& +\int_{S^{t-1}}-\int_{S^{i}} A_{S}^{i} \wedge|x|^{\alpha}\left(* F_{A}^{i}\right) d S \\
= & I_{1}+I_{2}+\text { boundary terms. }
\end{aligned}
$$

But

$$
I_{1} \leq \int_{U^{i}}|x|^{\alpha}\left|A^{i}\right||\varphi|^{2} d x \leq \int_{U^{i}}|x|^{\alpha}\left|A^{i}\right|^{2} d x+\int_{U^{i}}|x|^{\alpha}|\varphi|^{4} d x .
$$

Using Lemma $4.5\left(\mathrm{e}^{\prime}\right)$ of [8] we conclude that for any $\alpha>1$

$$
\int_{|x| \leq 1}|x|^{\alpha}\left|F_{A}(x)\right|^{2} d x \leq \int_{|x| \leq 1}|x|^{\alpha}|\varphi|^{4} d x+\kappa \int_{|x|=1}\left|F_{A}\right|^{2} d S
$$

where $\kappa$ is a positive constant. Writing (2.4) as a differental inequality we find that

$$
\int_{|x| \leq r}|x|^{\alpha}\left|F_{A}(x)\right|^{2} d x \leq C r^{\beta}
$$

with $\beta>0$ and $C, \beta$ independent of $\alpha$. Putting these estimates together we obtain

$$
|x|^{2} F_{A}(x) \leq C r^{\beta},
$$

from which the lemma follows by integration in spherical coordinates.

By Lemma 2.1, $\varphi$ is a weak solution in all of $B$. Lemma 2.3 implies that the curvature term $F_{A}$ in the second of inequalities (1.5) satisfies condition (5.13) of [2]. Using Theorem 5.3.1 of [2] we conclude that $|\varphi|$ is bounded on compact subdomains of $B$.

Lemma 2.3 also allows us to make a continuous gauge transformation to a gauge in which

$$
\left\{\begin{array}{l}
d^{*} A=0, \\
\|A\|_{H^{1, p}} \leq C\left\|F_{A}\right\|_{L^{p}}, \quad p=\frac{3}{2}+\varepsilon
\end{array}\right.
$$

for some $\varepsilon>0$. That this is possible follows from [11]. Following [10] we call any gauge in which (2.5) holds a Hodge gauge. In the remainder of the proof we assume that such a gauge has been fixed. 
LeMma 2.4. The connection $A$ is bounded in $B$.

Proof. Since $d^{*} A=0$ we can write

$$
\left(d^{*} d+d d^{*}\right) A=J(\varphi)-\frac{1}{2} d^{*}[A, A]-*\left[A, * F_{A}\right]
$$

in $B-\{0\}$ as a consequence of the first of eqs. (1.2). Then in this region the scalar $u=|A|+1$ satisfies

$$
-\Delta u \leq f(x) u, \quad \text { where } f=C\left(|\varphi|^{2}+|\nabla A|+\left|F_{A}\right|\right) .
$$

Applying Sobolev's inequality to (2.5) we find that

$$
u \in L^{(9+6 \varepsilon) /(3-2 \varepsilon)}(B) \text {. }
$$

Apply Lemma 2.1 with $q_{0}=(3+2 \varepsilon) /(6-4 \varepsilon)$ and $q=(9+6 \varepsilon) /(6-4 \varepsilon)$ to conclude that $u^{q}$ is a weak $H^{1,2}$ subsolution in $B$. In addition, since $f \in L^{p}(B)$ for some $p>n / 2$, Morrey's condition ((5.1.3) of [2]) is satisfied automatically (by integration in spherical coordinates and the Hölder inequality). Thus we apply Theorem 5.3.1 of [2] to conclude that $A$ is bounded on compact subdomains.

The proof of Theorem 1.2 is now completed as in [3] by invoking classical theorems of Hildebrandt-Widman and Schauder for bounded weak solutions of elliptic systems.

Acknowledgment. This research was partially supported by a grant from the United States Naval Academy Research Council.

\section{REFERENCES}

[1] N. Hitchen, Harmonic spinors, Adv. in Math., 14 (1974), 1-55.

[2] C. B. Morrey, Multiple Integrals in the Calculus of Variations, Springer, New York, 1966.

[3] T. H. Otway, Removable singularities in coupled Yang-Mills-Dirac fields, Comm. Partial Differential Equations, 12 (9) (1987), 1029-1070.

[4] T. H. Parker, Gauge theories on four dimensional Riemannian manifolds, Comm. Math. Phys., 85 (1982), 563-602.

[5] _- Conformal fields and stability, Math. Z., 185 (1984), 305-319.

[6] L. M. Sibner, Removable singularities of Yang-Mills fields in $\mathbb{R}^{3}$, Compositio Math., 53 (1984), 91-104.

[7] __ The isolated point singularity problem for the coupled Yang-Mills equations in higher dimensions, Math. Ann., 271 (1985), 125-131.

[8] L. M. Sibner and R. J. Sibner, Removable singularities of coupled Yang-Mills fields in $\mathbb{R}^{3}$, Comm. Math. Phys., 93 (1984), 1-17. 
[9] P. D. Smith, Removable singularities for Yang-Mills-Higgs equations in two dimensions, preprint.

[10] K. K. Uhlenbeck, Removable singularities in Yang-Mills fields, Comm. Math. Phys., 83 (1982), 11-29.

[11] Connections with $L^{p}$ bounds on curvature, Comm. Math. Phys., 83 (1982), $31-42$.

Received December 2, 1988 and in revised form March 13, 1989.

The University of Texas at Austin

AUSTIN, TX 78712 



\section{PACIFIC JOURNAL OF MATHEMATICS EDITORS}

\author{
V. S. VARADARAJAN \\ (Managing Editor) \\ University of California \\ Los Angeles, CA 90024-1555-05 \\ Herbert Clemens \\ University of Utah \\ Salt Lake City, UT 84112 \\ Thomas ENRIGHT \\ University of California, San Diego \\ La Jolla, CA 92093
}

\section{R. FINN}

Stanford University

Stanford, CA 94305

Hermann FlaschKa

University of Arizona

Tucson, AZ 85721

VAUGHAN F. R. Jones

University of California

Berkeley, CA 94720

STEVEN KeRCKHOFF

Stanford University

Stanford, CA 94305
C. C. MOORE

University of California

Berkeley, CA 94720

Martin ScharlemanN

University of California

Santa Barbara, CA 93106

HAROLd STARK

University of California, San Diego

La Jolla, CA 92093

\section{ASSOCIATE EDITORS}
R. ARENS
E. F. BECKENBACH
B. H. NeumanN
F. Wolf
K. YoshidA (1906-1982)
(1904-1989)

\section{SUPPORTING INSTITUTIONS}
UNIVERSITY OF ARIZONA
UNIVERSITY OF BRITISH COLUMBIA
CALIFORNIA INSTITUTE OF TECHNOLOGY
UNIVERSITY OF CALIFORNIA
MONTANA STATE UNIVERSITY
UNIVERSITY OF NEVADA, RENO
NEW MEXICO STATE UNIVERSITY
OREGON STATE UNIVERSITY
UNIVERSITY OF OREGON
UNIVERSITY OF SOUTHERN CALIFORNIA
STANFORD UNIVERSITY
UNIVERSITY OF HAWAII
UNIVERSITY OF TOKYO
UNIVERSITY OF UTAH
WASHINGTON STATE UNIVERSITY
UNIVERSITY OF WASHINGTON 


\section{Pacific Journal of Mathematics}

Vol. 146, No. $1 \quad$ November, 1990

Primo Brandi and Anna Salvadori, A quasi-additivity type condition and

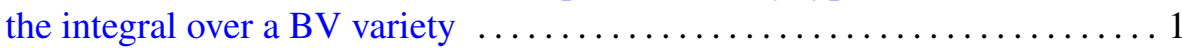

Dong M. Chung, Chull Park and David Lee Skoug, Operator-valued

Feynman integrals via conditional Feynman integrals ..............21

Paul Jolissaint, Index for pairs of finite von Neumann algebras . .........43

Miodrag Mateljević and Miroslav Pavlović, Multipliers of $H^{p}$ and

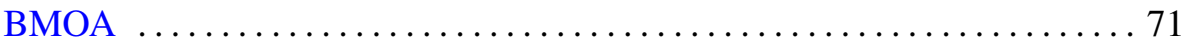

Himadri Kumar Mukerjee, Poincaré cobordism exact sequences and

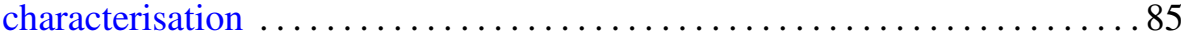

Thomas H. Otway, The coupled Yang-Mills-Dirac equations for differential

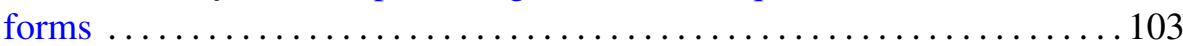

Sechiko Takahashi, Nevanlinna parametrizations for the extended

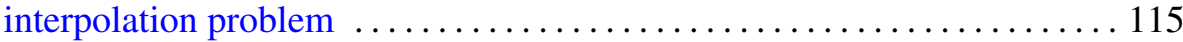

P. C. Trombi, Uniform asymptotics for real reductive Lie groups ........ 131 